\title{
Use of the Operon Structure of the C. elegans Genome as a Tool to Identify Functionally Related Proteins
}

\author{
Silvia Dossena Charity Nofziger Emanuele Bernardinelli Selma Soyal \\ Wolfgang Patsch Markus Paulmichl
}

Institute of Pharmacology and Toxicology, Paracelsus Medical University, Salzburg, Austria

\author{
Key Words \\ C. elegans $\cdot$ Operon $\cdot$ Nematode $\cdot$ Gene $\cdot$ Prediction $\cdot$ Protein-protein interaction $\cdot$ Protein \\ function
}

\begin{abstract}
One of the most pressing challenges in the post genomic era is the identification and characterization of protein-protein interactions (PPIs), as these are essential in understanding the cellular physiology of health and disease. Experimental techniques suitable for characterizing PPIs (X-ray crystallography or nuclear magnetic resonance spectroscopy, among others) are usually laborious, time-consuming and often difficult to apply to membrane proteins, and therefore require accurate prediction of the candidate interacting partners. High-throughput experimental methods (yeast two-hybrid and affinity purification) succumb to the same shortcomings, and can also lead to high rates of false positive and negative results. Therefore, reliable tools for predicting PPIs are needed. The use of the operon structure in the eukaryote Caenorhabditis elegans genome is a valuable, though underserved, tool for identifying physically or functionally interacting proteins. Based on the concept that genes organized in the same operon may encode physically or functionally related proteins, this algorithm is easy to be applied and, importantly, gives a limited number of candidate partners of a given protein, allowing for focused experimental verification. Moreover, this approach can be successfully used to predict PPIs in the human system, including those of membrane proteins.
\end{abstract}




\section{Introduction}

In the pre-genome era, the number of human genes coding for proteins was predicted to be around 100,000 [1]. However, in October 2004, the International Human Genome Sequencing Consortium revealed that the number of protein coding genes is only 20,00025,000 [2], a finding consistent with the fact that, in eukaryotes, the vast majority of nuclear DNA is non-coding [3]. The human gene count is, therefore, only slightly greater than that of organisms such as the roundworm (Caenorhabditis elegans) or the fruit fly (Drosophila melanogaster), which have about 20,000 [4] and 14,000 [5] genes, respectively. Apparently, this unexpectedly low number of coding genes cannot account for the complexity of the human organism, since it was previously thought that the number of genes reflects the phenotypic complexity of an organism. Since this concept is no longer valid, the question as to how a large number of different phenotypes can be generated by a limited number of genes is still pertinent. Importantly, phenotypic complexity can arise from a single protein interacting with several molecular partners, thereby leading to multiple functions. With this strategy, several biological functions can be determined by crosstalk between proteins organized in a network rather than by the characteristics of the individual components. Therefore the number of protein interactions in different organisms, rather than the number of their genes, appears to better correlate with their apparent phenotypic complexity. Indeed, Stumpf et al. estimated the number of protein-protein interactions (PPIs) in humans to be approximately 650,000, and found that the human protein interaction network (PIN) is one order of magnitude bigger than that of $D$. melanogaster, and three times bigger than that of C. elegans [6].

As PPIs are key to biological complexity, a detailed knowledge of the interactome [7] of a protein is crucial for understanding its physiology. There are two major ways to determine PPIs. One is through computational prediction and the other is by experimental detection. Experimental detection of PPIs is often laborious, time-consuming, difficult to apply to membrane proteins, and, with the exception of techniques that allow for high-throughput (HTP) screenings, needs a reliable prediction of the molecular partner(s) of a given protein. Therefore, there is an unmet need for the development of sensitive and user-friendly methods for predicting novel PPIs, as well as their cellular location and function. Such methods could be exploited for establishing differences in the interactome of a protein between a normal and diseased cellular state and provide insights into its pathophysiological role, thereby paving the way for novel therapeutic intervention and/or allowing identification of new pharmacological targets $[8,9]$.

Two interacting proteins can be associated on a molecular (i.e. the two partners establish a stable or transient direct contact) or functional level (i.e. the two partners belong to the same regulatory pathway or signaling cascade, without the need to interact physically). The establishment of a molecular interaction will likely lead to a new function, molecular partners are most likely also functional partners, but not vice-versa.

Here we suggest that the use of the operon structure present in the C. elegans genome could be used to predict functional and/or molecular interacting partners of a given protein-an extremely useful tool that can be applied to membrane proteins and translated to the human system.

\section{Computational prediction of PPIs based on genomic context and sequence}

Following the recent advances in complete genome sequencing, a gene is no longer regarded as a single and independent protein-coding entity. Only in the simplest cases does an individual gene code for a single protein or RNA. In general, genes consist of sequence modules that may combine to generate a coherent set of potentially overlapping functional products [10]. In addition, the context in which genes are organized in the genome can reveal potential functional interactions between their products, even if no sequence similarity 
between the genes exists [11-13]. Genomic context- and sequence-based methods (including the search for co-expressed and co-localized genes on chromosomes, Fig. 1) can also be used to detect the evolutionary fingerprints of interacting protein pairs, even in the absence of protein structure or primary sequence information.

\section{Co-expression of genes}

The physical interaction between two intracellular proteins is possible only if both are simultaneously expressed in the same cellular compartment. This concept is substantiated by the finding that protein pairs encoded by co-expressed genes (i.e. genes whose expression levels appear to be temporally correlated) interact with each other more frequently than those encoded by non-co-expressed genes $[14,15]$. Importantly, a large proportion of coexpressed and conserved gene pair products are either part of the same stable complex or physically interact [16]. Accordingly, positive correlations between the mRNA expression levels of interacting proteins in permanent functional complexes such as the ribosome and proteasome, are evident [17].

Gene co-expression can be achieved by overlapping transcription factor regulation. Another elegant way of co-expressing genes is via their organization into operons (Fig. 1a), a strategy that is often seen in prokaryotes. Genes arranged in this way are transcribed as a single unit or a polycistronic mRNA, under control of the same promoter. Indeed, a systematic comparison of a large number of prokaryotic genomes (bacterial and archaeal), revealed that proteins encoded by conserved gene pairs within operons appear to cooperate in a direct molecular interaction [18]. In eukaryotes, a similar genomic structure is less apparent and genes are usually transcribed individually. However, examples of polycistronic transcription are found (Fig. 1b), and can result in co-expression of functionally related proteins [19].

\section{Co-localization of genes}

If genes are situated in close genomic proximity (i.e. within 300 [20] to 500 base pairs [11]) and this genomic co-localization is conserved across several organisms (Fig. 1c), it can imply that these genes are involved in the same biological process/pathway [18]. Indeed, the analysis of conserved gene order allows for the identification of networks from pairwise genomic associations and may shed light on the functional relationships between the gene products [21].

\section{Operons in Caenorhabditis elegans}

Around $15 \%$ of all genes in C. elegans are arranged in operons or operon-like units, [22, 23 ] and represent a way by which co-localized genes may also be temporally and spatially co-expressed. A typical nematode operon is characterized by the presence of multiple coding sequences ( 2 to a maximum of 8 genes) at short intercistronic distances ranging between 50 and 200 base pairs [24], although distances as large as 2 kilobases have also been detected [25].

Genes within an operon (with the notable exception of hybrid operons [26]) are under the regulation of a single promoter at the $5^{\prime}$ end of the first gene, which leads to the transcription of a polycistronic pre-mRNA. Unlike the classical bacterial operon transcripts, those of the nematode are co-transcriptionally processed before ribosomal translation. The pre-mRNA molecule is processed in two steps: cis-splicing removes the introns, while transsplicing separates the single coding units and prepares the mRNA for translation. During trans-splicing, a Splice Leading sequence or Splice Leader (SL) is attached to the 5' end of the single coding mRNA. The $\sim 22$ nucleotide long SL is provided by a 100 nucleotide long small nuclear ribonucleoprotein (snRNP) [27]. SLs are needed for stabilization of the mRNA molecule in the absence of a capping sequence and allow for translation of the downstream genes in the operon, which lack the necessary recognition sequence for ribosomal loading. There are two main groups of SLs: SL1, almost always identical in sequence, and SL2 or 


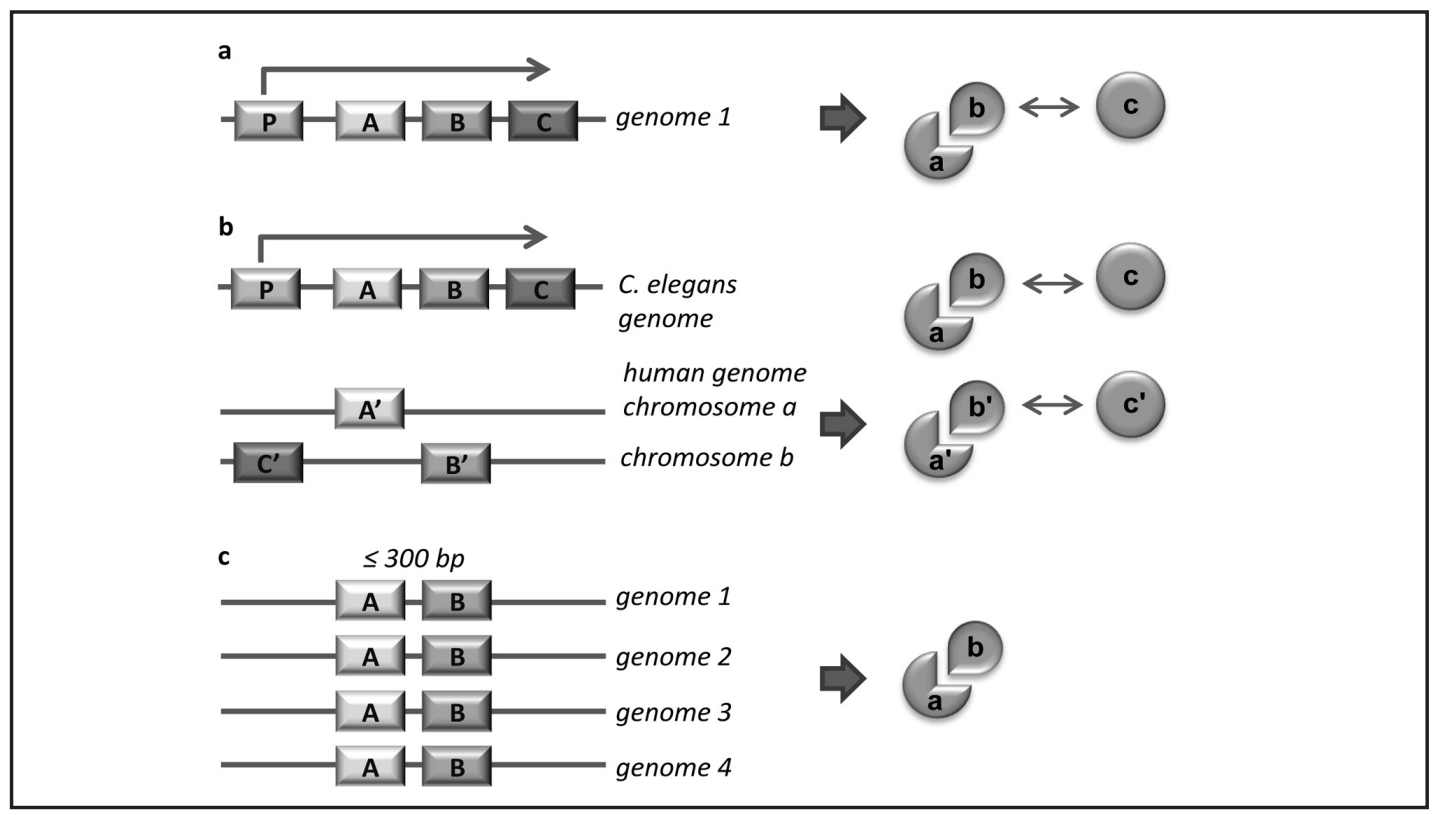

Fig. 1. Protein-protein interaction prediction methods based on genomic sequence. a, co-expression of genes. Genes A, B and C are organized in an operon. Consequently, they are transcribed under control of the same promoter (P) as a single unit and are temporally and spatially co-expressed. These genes may potentially encode for physically (proteins a and b) or functionally (proteins b and c; the functional interaction is represented as a double arrow) related proteins. b, operon- $\underline{b}$ ased partner protein quest (OBPQ). As shown in a, genes A, B and C are organized in an operon, as occurs in the eukaryote $C$. elegans. This organization allows for the prediction of functional and/or molecular interactions between the proteins (a', b' and c'; the functional interaction is represented as a double arrow) encoded by the respective human orthologue genes A', B' and C', even if their spatial arrangement is not conserved in the human system. c, co-localization of genes. Orthologues of genes A and B are repeatedly observed in close proximity across the genome of several organisms. Hence, it is possible to predict a functional or physical interaction between the protein products ( $\mathrm{a}$ and $\mathrm{b}$ ) of genes $\mathrm{A}$ and $\mathrm{B}$.

SL2-like, which show higher sequence variability [23]. Whereas SL1 is usually placed on the transcript of the first gene within an operon, SL2 or SL2-like sequences are usually trans-spliced on the transcript(s) of downstream gene(s). After this, the processed mRNAs harboring the new SL2 or SL2-like sequences at the 5' end are translated into the relative gene products.

SL1 sequences are not only added onto transcripts of genes leading an operon. These special signals are also found on transcripts of genes that do not belong to operons, as well as on transcripts of genes that are located downstream of an operon unit. Examples of the latter include hybrid operons, that possess internal promoters in addition to the $5^{\prime}$ promoter, and "alternative operons", that lack the intercistronic sequence [28]. Therefore, SL1 transsplicing cannot be used to identify genes organized in operons or predict their position within an operon [23]. In contrast, detection of SL2 or SL2-like sequences on a transcript is strongly indicative that the respective gene is contained within an operon, but is not unequivocal proof, since SL2 trans-splicing can occasionally occur at cis-splice sites of genes not embedded in operons [23]. In addition, there are some rare examples where SL2 trans-splicing occurs in a gene that is in the first position of an operon. Because of these ambiguities in terms of SL1 or SL2 sequences, all putative operons listed in WormBase (http://www.wormbase. org/) were re-categorized on one consistent criterion: an SL2 or SL2-like trans-spliced transcript with a frequency higher than $5 \%$ of the total counts of trans-splicing events for that specific transcript (the rest of the events being SL1-trans-splicing) had to be detected in order for the particular gene to be identified as part of an operon [29]. This study not only 
supported previous estimates that $\approx 70 \%$ of $C$. elegans genes are trans-spliced and that $>17 \%$ are organized in operons, it also refined the previous knowledge of operon organization [23, 29]. The arbitrary cut-off value of 5\%, however, bears the possibility that the real number of operons might be underestimated, since low levels of co-transcription with the upstream gene were not accounted for. Therefore, the only unequivocal way to demonstrate the existence of an operon is by the identification of a polycistronic pre-mRNA-a process which can be difficult since pre-mRNAs are rapidly processed [30].

The genes encoded in C. elegans operons appear to be heterogeneous and, in contrast to what is often seen in bacterial operons, do not code for all the proteins involved in a single biochemical pathway (e.g. the lac operon in E. coli) [31]. Some gene families also appear to be more often represented in operons than others, such as constitutive and ubiquitously expressed genes, as well as those required for rapid growth, involved in RNA processing and translation, or those coding for mitochondrial proteins [28]. In addition, approximately one quarter of C. elegans operons have internal promoters, leading to different levels and patterns of expression of the genes they regulate [26]. On the other hand, tissue specific genes, which require local regulation in response to highly specific stimuli, are poorly represented in operons. However, many gene products encoded by the same operon in C. elegans have been shown to be linked by a common function or expression pattern, and are often involved in the same pathway or physically interact with each other [24]. This organization allows the genome to be condensed into compact, co-regulated units. Phylogenetic analyses show a high conservation of some operons throughout the entire nematode phylum [32], clearly indicating that loss of such genomic organization would sacrifice the efficient co-expression of essential gene products and would therefore not be evolutionarily advantageous.

The Operon-Based Partner Protein Quest (OBPQ): the use of the operon structure in $C$. elegans for predicting/identifying novel PPIS

As previously mentioned, the relative proximity of genes to one another, as well as their co-expression (Figs. 1a-c), are useful features for predicting possible functional interactions between proteins of interest; the operon structure of the C. elegans genome can be considered an ideal example of genomic co-localization that allows both the co-regulation and co-expression of a number of genes in an efficient way. If the hypothetical genes $\mathrm{A}, \mathrm{B}$ and $\mathrm{C}$ are organized in the same operon, they are transcribed under control of the same promoter as a single unit and are consequently temporally and spatially co-expressed (Fig. 1b). Hybrid operons, whose genes can be transcribed independently due to the presence of internal promoters, represent notable exceptions to this rule. In addition, genes encoding constitutively expressed proteins may be under the control of the same constitutive promoter. In both of these circumstances, a molecular or functional relationship between the proteins of genes contained in the same operon is unlikely. However, another intriguing option is that genes belonging to the same operon are temporally and spatially co-expressed because the proteins encoded by them are functionally and/or molecularly related [33].

Since the genome of $C$. elegans is fully sequenced with many operons established, inspection of the list of the genes organized in operons [22,23] provides information about the potential molecular or functional partner(s) of a given protein. Indeed, gene products encoded by the same operon and displaying an obvious functional relationship have been retrospectively identified in different functional modules of $C$. elegans (RNA processing, longevity regulation, collagen modification, protein degradation, morphogenesis, ion transport, etc.) [24]. We used, for the first time, the operon structure of C. elegans to prospectively predict PPIs [33]. Importantly, once a putative PPI identified by the operon structure is demonstrated to occur in C. elegans, an evolutionary conserved PPI can be further predicted by identifying the orthologues of the respective interacting proteins in the human system. We firstly and successfully applied such a strategy, which we subsequently defined as the operon-based partner protein quest (OBPQ) [34]. To make this idea more tangible, consider the previous example where the proteins $\mathrm{a}, \mathrm{b}$ and $\mathrm{c}$ are encoded by genes A, B and C organized in a C. elegans operon (Fig. 1b) and are predicted to functionally and/ 
or molecularly interact in this organism. Since the molecular physiology of C. elegans is to some extent similar to that in humans, it is feasible to expect that interacting proteins in the nematode interact in higher organisms as well. Let us assume that the nematode genes $\mathrm{A}, \mathrm{B}$ and $\mathrm{C}$ and the human genes $\mathrm{A}^{\prime}, \mathrm{B}^{\prime}$ and $\mathrm{C}^{\prime}$ are orthologues. The OBPQ concept allows the prediction of functional and/or direct molecular interactions between the proteins $\mathrm{a}^{\prime}, \mathrm{b}$ ' and c' encoded by genes A', B' and C' in human, even though the spatial neighborhood of these genes may not be conserved (Fig. 1b) [34]. In addition, if one of the proteins encoded by genes embedded in an operon has a well-established function, it is possible that the other proteins within the same operon belong to the same functional module [35].

Importantly, the OBPQ also includes predictions of functional partners for membrane proteins that are inherently difficult to make with other methods. Examples of $C$. elegans ion channel genes contained within operons that are reported in WormBase or gene pairs that are located at an intercistronic distance of $\leq 2500$ base pairs, along with the corresponding human orthologues, are listed in Table 1 (an extended version of this table is provided at: http://www.pmu.ac.at/fileadmin/user_upload/files/PDF/1311_Pharma_Table_1.pdf). This table is an updated version of the one we previously compiled [34] and should prompt the reader to easily verify if the operon structure of the $C$. elegans genome suggests a potential functional and/or molecular partner of a given membrane protein. As an example, consider the human potassium channel Kv1.1. The orthologue of the gene encoding Kv1.1 in C. elegans is $s h k-1$. No genes are located within a short $(\leq 2500 \mathrm{pb})$ intercistronic distance with respect to shk-1 in the C. elegans genome, therefore the OBPQ approach described cannot be applied for predicting molecular partners of Kv1.1.

Let us now consider the human potassium channel Kv-beta-1 (Table 1), encoded by the gene KCNAB1, a C. elegans orthologue of which is kvs-4. kvs4 is embedded in an operon (CEOP3712) with exos-1, that encodes a protein whose human orthologous is EXOSC1/CSL4. In this case, the OBPQ predicts that a functional and/or molecular interaction between Kvbeta-1 and EXOSC1/CSL4 may occur in humans. The $C$. elegans orthologue of each human gene in Table 1, the neighboring or operon partner gene(s) and the corresponding human orthologue(s) were retrieved using WormBase (http://www.wormbase.org/).

In the following, only those cases where a functional and/or molecular interaction between two proteins predicted by the OBPQ was verified on an experimental level or can be envisioned based on experimental evidence are discussed. For example, the C. elegans gene encoding for the orthologue of the human cardiac muscle ryanodine receptor-calcium release channel (RYR-2) is unc-68 (Table 1, calcium channels). unc-68 is located within a relatively short genomic distance $(\approx 2060 \mathrm{bp})$ from odc-1, the human orthologue of which is ornithine decarboxylase $\underline{1}$ (ODC1) - a rate-limiting enzyme in polyamine biosynthesis.

Table 1. Membrane proteins encoded by genes organized in operons or located at an inter-cistronic distance $<2500$ base pairs in C. elegans and the corresponding human orthologues. A number of different classes of human membrane proteins (ion channels) with their relative UniProt accession number (Acc\#) and gene name are reported in the first column (Human). The C. elegans orthologs of each human gene was retrieved consulting WormBase (http://www.wormbase.org/, last access: October 2013). The orthologs in $C$. elegans and their established or putative operon partner genes (next gene) are listed in the second column (Nematode), and the approximate intercistronic distance is also given for upstream (u) or downstream (d) genes located within 2500 base pairs. If available, the relative reference number and name for each nematode gene are reported. The nematode genes for which a channel-forming function of the corresponding protein is established are marked with $\left(^{\circ}\right)$. The third column (Human) shows the corresponding human orthologues of the nematode genes listed as "next gene", with their specific Acc\#, names and a brief functional description. In the right column, the references demonstrating a functional or physical interaction between the human gene product pairs listed in the first and third column are indicated. A question mark (?) indicates that the interaction could potentially be extrapolated to humans, but was shown in another organism. An extended version of this table is provided at: http://www.pmu.ac.at/fileadmin/user_upload/ files/PDF/1311_Pharma_Table_1.pdf. 


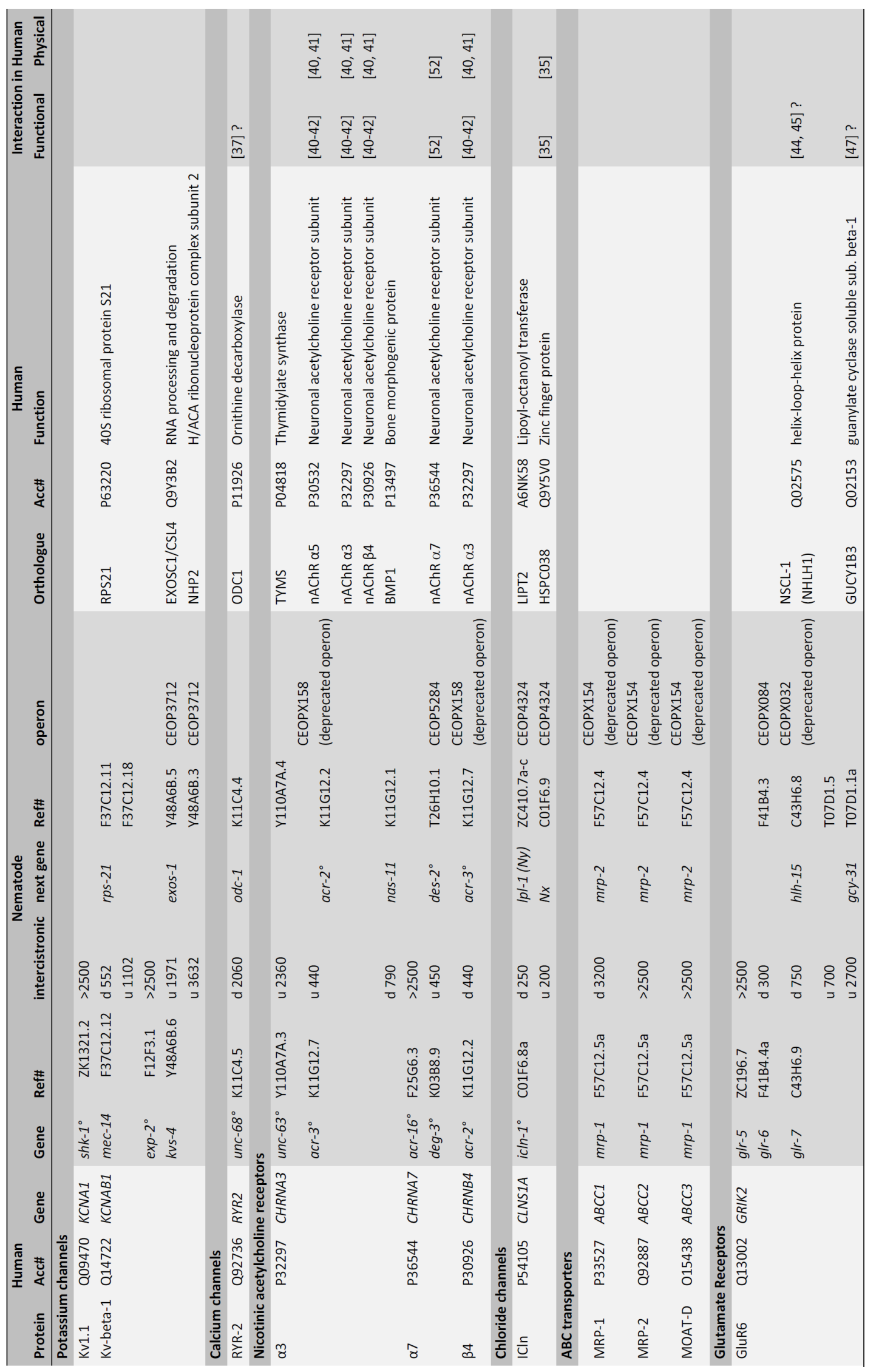


Although WormBase does not predict that unc- 68 and odc- 1 belong to the same operon, the relatively short genomic distance suggests a hypothetical functional and/or molecular relationship between their human orthologues (RYRs and ODC1). Endogenous polyamines, in particular spermine, have been found to block and/or modulate a number of ion channels [36], including RYR from adult rabbit ventricular muscle [37]. Consequently, it can be envisioned that the expression level and activity of ornithine decarboxylase could affect the biophysical properties of RYR-2. Evidence exists that this functional link could be extended to other isoforms of RYR, such as the skeletal muscle isoform [38], at least in rabbit. It is important to note that this scenario has not been experimentally confirmed.

Expression studies in Xenopus oocytes demonstrated that a functional neuronal nicotinic acetylcholine receptor (nAChr) of $C$. elegans is composed of three $\alpha$-subunits (UNC-38, UNC63 and ACR-12) and two non- $\alpha$ subunits (ACR-2 and ACR-3). Although this receptor complex exhibits a partially overlapping subunit composition with the muscle acetylcholine receptor, it shows distinct pharmacology that is important in controlling the excitability of cholinergic motor neurons [39]. These data demonstrate an obvious functional relationship between the $a c r-2$ and $a c r-3$ gene products in the nematode. $a c r-3$ is orthologous to the human nAChr $\alpha 3$ subunit, while acr- 2 is orthologous to the human nAChr $\beta 4, \alpha 3$ or $\alpha 5$ subunits (Table 1 , nicotinic acetylcholine receptors). Notably, human nAChr $(\alpha 3)_{2}(\beta 4)_{3}$ and $(\alpha 3)_{2}(\beta 4)_{2} \alpha 5$ represent the ganglion-type nicotinic receptors located in the autonomic ganglia [40, 41], and substantiate the functional and molecular relationship between human orthologues of nematode genes located at relatively short intercistronic distances. The human genes encoding the nAChr $\alpha 3, \beta 4$ and $\alpha 5$ subunits lie in a genomic cluster, and may have a role in nicotine addiction and lung cancer [42]. Indeed, a short intercistronic distance $(\approx 440$ bp) separates acr-2 and $a c r-3$ in the nematode genome (Table 1). Again, WormBase does not identify acr-2 and acr-3 as belonging to the same operon, as the extent of SL2 transsplicing of the $a c r-3$ transcript does not reach the suggested cut-off value of $5 \%$. However, using RT-PCR, we identified SL1 and SL2 sequences in the gene transcripts of acr-2 and acr-3, respectively [34]. Using this approach, we also showed that the transcripts of two members of the ABC superfamily of transport proteins, $m r p-1$ and $m r p-2$, show SL1 and SL2 sequences, respectively (Table $1, \mathrm{ABC}$ transporters). Again, WormBase does not identify the latter gene couple as members of the same operon. It is important, however, as stated above, that the sole presence of SL sequences does not prove that the respective genes belong to an operon. Only the identification of the respective polycistronic pre-mRNA would unambiguously confirm that fact.

Similarly, the nematode genes glr-7, hlh-15, T07D1.5 and gcy-31 are also separated by short intercistronic distances (Table 1 , glutamate receptors), and have been predicted to lie within the same operon. $h l h-15$ encodes a basic domain helix-loop-helix (HLH) transcription factor. The human orthologue of glr-7 is GluR6, a subunit of the kainate-type glutamate receptor, and the human orthologue of hlh-15, NSCL-1 (NHLH1), has been identified as a DNA binding protein involved in cell type determination in the developing nervous system [43]. In mice, expression of both GluR6 and NSCL-1 is controlled by HLH transcription factors encoded by the Neurogenin 1 and 2 genes [44, 45], which play a key role in the specification of the neuronal phenotype and control differentiation of neocortical neurons. The fact that both GluR6 and NSCL-1 are regulated by the same transcription factors in mammals is a clue that they may functionally interact, at least in mouse. The human orthologue of gcy-31 is GUCY1B3, an isoform of guanylate cyclase soluble subunit beta-1. Activation of kainate-type glutamate receptors regulates increases in intracellular calcium levels. Specifically, calcium binds to calmodulin and results in the activation of neuronal nitric oxide (NO) synthase. NO, in turn, stimulates soluble guanylate cyclase, and increases the production of cyclic guanosine monophosphate (cGMP) [46, 47]. This signal transduction cascade modulates cerebral processes such as circadian rhythms, the sleep-waking cycle, learning and memory $[48,49]$. Therefore, it seems that the mammalian orthologues of glr-7, hlh-15 and gcy-31 C. elegans genes are functionally related. The putative function and human orthologue of T07D1.5 is currently unknown. Whether or not these genes are truly organized together in 
an operon, and whether a possible functional interaction of the respective gene products can be transposed to the human system requires further investigation.

Examples of nematode plasma membrane proteins encoded by genes embedded in the same operon (according to WormBase, see Table 1) and, most importantly, with an experimentally validated functional interaction, are the subunits of acetylcholine receptors encoded by des-2 and deg-3 [50], as well as the splicing variants of the nucleotide-sensitive chloride current protein, ICln (IClnN2), with the zinc-finger protein, HSPC038 [24, 33, 34]. deg-3 (Table 1, nicotinic acetylcholine receptors) encodes an $\alpha$ subunit of the neuronal nAChR sodium channel, and was originally identified by a gain-of-function mutation, u662, which resulted in neuronal degeneration, presumably by deregulation of channel activity. deg-3 mRNAs possess SL2 trans-spliced 5' ends, suggesting that the gene is co-transcribed with at least one other gene in the same operon [51]. It was later demonstrated that des2 , which also encodes a nAChR subunit, is indeed the upstream gene located in the same operon with deg-3. des-2 and des-3 have similar expression patterns in C. elegans, and only the co-translation of both their corresponding complementary (c) RNAs (but not the single cRNAs) resulted in a functional acetylcholine-gated sodium channel in Xenopus oocytes. Moreover, mutations in des-2 prevented deg-3(u662)-induced degenerations, further suggesting that des-2 is needed for the deg-3(u662) phenotype in vivo. These results show that the operon structure regulates the coordinate expression of the two channel subunits [50]. des-2 and deg-3 are orthologous to the human nAChr $\alpha 7$ subunit (Table 1) that can form homo-pentameric receptors [52].

Recently, we showed that the molecular and functional interaction between the nematode proteins IClnN2 and Nx is conserved in humans [35]. This represents a further example of the usefulness of the OBPQ tool in defining PPIs, and will be discussed in detail below.

The molecular and functional interaction between ICln and HSPCO38

ICln is an ubiquitously expressed, multifunctional protein $[7,53]$ essential for cellular and embryonic viability [54]. It was first characterized as a chloride channel activated during regulation of cellular volume induced by hypotonic challenge [55]. Despite being located mainly in the cytosol, ICln transposes towards the plasma membrane after cell swelling [56], where it participates in the activation of the swelling-induced chloride current (IClswell) an essential player in fundamental cellular processes including regulatory volume decrease (RVD), migration, proliferation and apoptosis [57]. In an attempt to better understand the physiological role of this essential protein, a number of studies were started with the aim of characterizing its interactome $[7,58,59]$. For this purpose, the operon structure of the $C$. elegans genome was used. In C. elegans, two splice variants of ICln (IClnN1 and IClnN2) are expressed. The open reading frame (ORF) of IClnN1 (GenBankTM/EBI Data Bank accession number AF202931) is composed of 618 base pairs (including the stop codon) and codes for a protein of 205 amino acids. The ORF of IClnN2 (accession number AF202932) is composed of 678 base pairs (including the stop codon) and codes for a 225 amino acid long protein [33]. Analysis of the IClnN1 and IClnN2 mRNAs using 5'-RACE revealed the presence of SL2-like sequences in the $5^{\prime}$-untranslated regions, indicating that the nematode ICln gene might be embedded in an operon. As mentioned earlier, SL2 and SL2-like sequences are usually found on monocistronic mRNAs encoded by genes organized in operons, but not in the lead position of the operon [60]. Analysis of the genomic sequence upstream and downstream of the nematode ICln gene led to the identification of two ORFs, Nx (222 base pairs, GenBankTM/EBI Data Bank accession number AF202929) and Ny (330 base pairs, accession number AF202930), respectively (Table 1, chloride channels). Both ORFs contain only 3 exons, and are transcribed in a polycistronic complex with ICln. No additional genes upstream of the Nx ORF were identified, indicating that it is the first gene of the operon, and SL1 sequences were identified only on the Nx transcript. Moreover, one SL2 sequence and three additional SL2-like sequences were identified in the Ny transcript, and no other coding regions were found downstream of Ny using 3'-RACE. 
The C. elegans proteins IClnN2 and Nx establish a functional interaction. As mentioned earlier, two splice variants of ICln mRNA, IClnN1 and IClnN2, are expressed in nematodes. The amino acid sequence of IClnN1 is orthologous to the ICln sequences identified in different species. The second splice variant (IClnN2) is characterized by an additional 60 base pair exon (exon 2a) located between exons 2 and 3, which code for a string of 20 amino acids (VRRRRRAPVLRTIQEDDEQR, single letter amino acid code). The additional amino acid string encoded by exon $2 \mathrm{a}$ is unique for the nematode IClnN2 sequence and is not found in any other organism. Functional reconstitution of recombinant purified IClnN1 in microscopic artificial lipid bilayers (tip-dip technique [61]) revealed channel activity with a small single channel conductance and open probability $\left(\mathrm{P}_{\mathrm{o}}\right)$ independent from the holding potential. The single channel conductance of IClnN2 was not different from that of IClnN1; in contrast, its $\mathrm{P}_{0}$ showed a strong dependence on the applied voltage, dropping to values near zero at positive holding potentials. This peculiar behavior was ascribed to the presence of the amino acids encoded by exon 2a. Importantly, the voltage-dependent inactivation at positive holding potentials observed after reconstitution of IClnN2 was markedly reduced in the presence of the Nx protein [33]. Provided that only the two purified proteins (IClnN2 and $\mathrm{Nx}$ ) were present in the system for the in vitro reconstitution of ion channels used in these experiments, the modification of electrophysiological properties of IClnN2 observed can be attributed to a direct molecular interaction between the two proteins, thus confirming that genes belonging to the same operon in C. elegans can encode proteins that establish a direct molecular interaction and/or are functionally linked $[19,62]$.

The human orthologs of the C. elegans ICIn and Nx (HSPC038) establish a direct molecular interaction. The human ortholog of the nematode protein Nx is HSPC038 (human stem progenitor cells 038). In contrast to the nematode system, the human ICln (CLNS1A) and HSPCO38 genes are not in close genomic proximity to each other (the ICln gene is located at position 11q13.5 whereas the HSPCO38 gene is located at position 8q22.3). Organization of the two nematode orthologs in the same operon led to the hypothesis that the CLNS1A and HSPC038 protein products are functionally linked. Indeed, a direct molecular interaction between human ICln and HSPC038 was found in vivo using fluorescence resonance energy transfer (FRET) [34]. This binary interaction was confirmed by in vitro binding assays, affinity chromatography and nuclear magnetic resonance (NMR) spectroscopy, which allowed for the characterization of the interaction interfaces and identification of the residues involved in formation of the complex [35].

Function of the ICIn/HSPCO38 complex. Experimental confirmation of the predicted ICln/ HSPC038 interaction allowed for the functional testing of the physiological role of HSPC038, whose purpose was previously unknown. Based on the knowledge that ICln is involved in RVD, it was inferred that HSPC038 might also belong to the same functional module. Indeed, western blot and in vivo functional testing by patch clamp and FRET showed that the ICln/ HSPC038 interaction is increased following hypotonic stress. The formation of the complex facilitates the translocation of ICln towards the plasma membrane and the activation of IClswell [35]. These results show that the operon structure of the C. elegans genome can be an extremely useful tool for predicting unknown interacting partners of a protein, including those of the plasma membrane. Importantly, the prediction can be successfully transposed to the human system and may allow for identification of the function of apparently unrelated proteins. The possible functional/molecular relationship between ICln and Lipoylligase B, the human orthologue of $\mathrm{Ny}$, is currently under investigation.

\section{Poly-cistronic gene organization in eukaryotes besides nematodes}

The organization of genes in operons seems also to be adopted by other eukaryotic organisms; for example, recent studies showed that the D. melanogaster genome contains gene couples that are transcribed as bicistronic mRNA molecules under the regulation of a single promoter upstream of the first gene. In the presence of bicistronic mRNA, the 
expression of the downstream gene is not regulated by a trans-splicing mechanism as in the nematode, but instead appears to be achieved via either internal ribosome entry sites (IRES), leaky ribosomal scanning, re-initiation mechanisms, or post-transcriptional processing [63, 64]. A global analysis of the transcripts produced in Drosophila predicted the existence of about 100 putative bicistronic transcripts bearing the necessary features for co-expression. A number of possible interesting interaction couples have therefore been identified, making a detailed analysis of such an expression mechanism as worthy as that previously described for $C$. elegans. Even though the identity of the protein product(s) from a bicistronic unit are not always known, the evidence that they are co-regulated on a transcriptional and translational level is a justifiable hint for a possible interaction between the respective gene products, either by direct binding or involvement in the same cellular pathway or regulatory issue [64]. A specific example in this context is represented by the CheB42a/llz locus. The D. melanogaster Degenerin/epithelial $\underline{\mathrm{Na}}^{+}$channel (Deg/ENaC) gene lounge lizard (llz) is co-transcribed in an operon-like locus with another gene of unknown function, CheB42a. $\mathrm{Deg} / \mathrm{ENaC}$ represent a diverse family of voltage-insensitive cation channels whose functions include transepithelial $\mathrm{Na}^{+}$transport, mechano-sensing, nociception, salt sensing and neurotransmission. CheB42a is a non-homologues gene located $<100$ base pairs upstream of llz. The two genes are transcribed from a single promoter as one primary transcript and are processed post-transcriptionally to generate individual mRNAs. The organization of the CheB42a/llz locus is conserved across Drosophila species; moreover, CheB42a and llz show similar expression patterns [63]. These observations point to a functional or molecular relationship between the two respective protein products, LLZ and CHEB42A. Indeed, when co-expressed, LLZ co-precipitated with CHEB42A, suggesting that the two proteins form a complex. Although LLZ expressed either alone or with CHEB42A did not generate ion channel currents, CHEB42A increased current amplitude of the Deg/ENaC acid-sensing ion channel 1a (ASIC1a) [65]. Another example of a bicistronic transcript in D. melanogaster is Tektin-C/CG10542 and cyclin- dependent kinase $\underline{5}$ (Cdk5)/flotillin. The functions of Tektin-C and CG10542 encoded proteins are unknown, but they may be involved in cytoskeleton organization. Flotillin is an integral membrane protein and Cdk5 is a soluble protein. Both flotillin and Cdk5 contribute to neuronal signal transduction and have been implicated in the pathogenesis of Alzheimer's disease in humans [63]. Therefore, operon-like organization and transcription may be more common in eukaryotes than previously appreciated and serve as a precious source of information for detecting novel PPIs.

\section{Poly-cistronic transcripts in humans}

The existence of functional polycistronic, operon-like structures, similar to those found in D. melanogaster, have also been found in Homo sapiens. The mechanisms allowing for the expression of bicistronic genes, even though not fully understood, appear to be similar to those already mentioned for Drosophila, i.e. IRESs and/or translation re-initiation. The characterization of the growth and differentiation factor 1 (GDF-1) protein by Lee in 1991 provided the first documentation of a human bicistronic structure [66]. GDF-1 is a transforming growth factor-beta family member originally isolated from a mouse embryo cDNA library, is expressed specifically in the nervous system and functions in left-right patterning of the mouse embryo. An additional ORF around 300 base pairs upstream of the initial GDF-1 start codon was identified. The newly identified ORF (named UOG, upstream of GDF-1) encoded a protein later characterized as ceramide synthase 1 (CERS1, LASS1), an orthologue of the S. cerevisiae longevity assurance gene 1 (LAG1) [67]. GDF-1 and CERS1 are translated as two independent proteins from a bicistronic mRNA. No functional interaction between GDF-1 and CERS1 has been demonstrated so far, nevertheless, the bicistronic structure seems to be conserved in mammals. Interestingly, the GDF-1/LASS1 transcript is strongly upregulated in the inflamed colonic mucosa of patients with Crohn's disease [68]. 
Another example of a mammalian bicistronic transcript was described by Gray et al. [69] and includes the ORF encoding the small nuclear ribonucleoprotein $\underline{N}$ (SNRPN). SNRPN

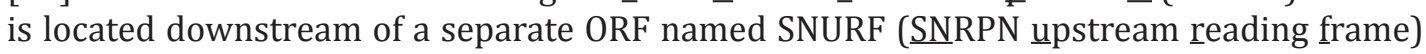
and encodes the spliceosomal protein SmN. SmN is involved in the etiology of Prader-Willi Syndrome (PWS), a developmental and neurobehavioral disorder resulting from the loss of function of genes in the chromosomal region 15q11-q13 [70]. SNURF encodes a highly basic, 71 amino acid long protein targeted to the nucleus that has also been implicated in PWS pathology. SNRPN and SNURF are independently expressed in the same cell type and both are absent in patients with PWS, enforcing the hypothesis that the bicistronic transcript may play a role in the development of the disease [69].

In human liver, Reiss et al. [71] identified an apparent bicistronic structure including the coding sequences of molybdenum cofactor synthesis-step 1A and B (MOCS1A and MOCS1B), the first two enzymes in the synthesis pathway of molybdopterin. Molybdopterin is a molybdenum-complexing factor necessary for the activation of molybdenum-dependent enzymes, such as xanthine dehydrogenase and sulphite oxidase [72]. Besides the bicistronic structures described so far, which are translated into separate polypeptides, no in vivo evidence for the independent translation of MOCS1A and MOCS1B has been found [73]. A further analysis of the genetic locus coding for the two proteins revealed an additional splicing site at the 3' of MOCS1A that could bypass its normal termination nonsense codon. This would result in union of the MOCS1A and MOCS1B ORFs and lead to translation of the two polypeptides into a single bifunctional protein [74]. This last example describes an extreme mechanism of co-expression and co-regulation, where two functionally linked proteins are fused into a single multifunctional polypeptide.

\section{Experimental methods to identify and characterize PPIs}

Any PPI identified by computational prediction needs subsequent experimental verification.

Yeast two-hybrid and Affinity Purification-Mass Spectrometry are two HTP methods by which PPIs can be experimentally identified. It is important to mention, however, that the HTP methods often utilize engineered proteins in a heterologous expression system, and can carry a considerably high amount of false positive results. Even though they are experimentally based, these approaches should be considered predictive, and therefore need further verification.

Experimental methods capable of confirming and characterizing molecular PPIs predicted by the computational and HTP experimental methods described above include X-ray crystallography, Nuclear Magnetic Resonance spectroscopy, surface plasmon resonance, atomic force microscopy, electron microscopy, co-immunoprecipitation and FRET. These methods can provide precise characterization of PPIs, but are scarcely suitable for HTP screenings or for the identification of unknown partners of a given protein, and are often difficult to apply to membrane proteins. Consequently, a reliable prediction of potential binding partners is needed prior to experimental validation.

\section{Conclusion}

The operon structure of the C. elegans genome can be an extremely useful tool to predict unknown interaction partners of a protein, including those of integral plasma membrane proteins. Indeed, C. elegans genes embedded in operons may encode proteins with unrelated sequence but related function. Since operons contain a limited number of genes, the OBPQ will give a limited number of candidate partners for a given protein, allowing for focused verification with experimental techniques. Despite this approach may seem to be straightforward, it was largely underserved so far. A further outcome of OBPQ 
is that knowing the function of one protein may allow for predicting the function of other proteins encoded by genes embedded in the same operon. Importantly, this approach can be successfully translated to the human system. This concept may allow for prediction and identification of the function of proteins that otherwise would not be apparent.

In summary, even though the functional relationship between gene products encoded in a same operon is not always obvious, in a number of cases it indicates an involvement of the relative human orthologues in a common pathway or regulatory issue and may be an extremely useful tool for identifying novel nodes of interaction in the intricate network of PPIs. The OBPQ concept may suggest unexplored direct molecular interactions and/or functional relationships difficult or impossible to predict with other tools.

\section{Conflict of Interest}

The authors have no conflict of interest to disclose.

\section{Acknowledgments}

We would like to sincerely thank Tom Blumenthal for helpful discussions. C.N. is supported by the Roche Postdoc Fellowship Program (\#231). This work was further supported by the FWF (P18608) and FP-7 (PIRSES-GA-2008-230661) grants to M.P. The authors acknowledge the expert secretarial assistance of Elisabeth Mooslechner.

\section{References}

1 Liang F, Holt I, Pertea G, Karamycheva S, Salzberg SL, Quackenbush J: Gene index analysis of the human genome estimates approximately 120,000 genes. Nat Genet 2000;25:239-240.

Consortium IHGS: Finishing the euchromatic sequence of the human genome. Nature 2004;431:931-945.

Gregory TR: Synergy between sequence and size in large-scale genomics. Nat Rev Gen 2005;6:699-708.

Consortium TCeS: Genome sequence of the nematode C. elegans: A platform for investigating biology.

Science 1998;282:2012-2018.

5 Adams MD, Celniker SE, Holt RA, Evans CA, Gocayne JD, Amanatides PG, Scherer SE, Li PW, Hoskins RA, Galle RF, George RA, Lewis SE, Richards S, Ashburner M, Henderson SN, Sutton GG, Wortman JR, Yandell MD, Zhang Q, Chen LX, Brandon RC, Rogers YH, Blazej RG, Champe M, Pfeiffer BD, Wan KH, Doyle C, Baxter EG, Helt G, Nelson CR, Gabor GL, Abril JF, Agbayani A, An HJ, Andrews-Pfannkoch C, Baldwin D, Ballew RM, Basu A, Baxendale J, Bayraktaroglu L, Beasley EM, Beeson KY, Benos PV, Berman BP, Bhandari D, Bolshakov S, Borkova D, Botchan MR, Bouck J, Brokstein P, Brottier P, Burtis KC, Busam DA, Butler H, Cadieu E, Center A, Chandra I, Cherry JM, Cawley S, Dahlke C, Davenport LB, Davies P, de Pablos B, Delcher A, Deng Z, Mays AD, Dew I, Dietz SM, Dodson K, Doup LE, Downes M, Dugan-Rocha S, Dunkov BC, Dunn P, Durbin KJ, Evangelista CC, Ferraz C, Ferriera S, Fleischmann W, Fosler C, Gabrielian AE, Garg NS, Gelbart WM, Glasser K, Glodek A, Gong F, Gorrell JH, Gu Z, Guan P, Harris M, Harris NL, Harvey D, Heiman TJ, Hernandez JR, Houck J, Hostin D, Houston KA, Howland TJ, Wei MH, Ibegwam C, Jalali M, Kalush F, Karpen GH, Ke Z, Kennison JA, Ketchum KA, Kimmel BE, Kodira CD, Kraft C, Kravitz S, Kulp D, Lai Z, Lasko P, Lei Y, Levitsky AA, Li J, Li Z, Liang Y, Lin X, Liu X, Mattei B, McIntosh TC, McLeod MP, McPherson D, Merkulov G, Milshina NV, Mobarry C, Morris J, Moshrefi A, Mount SM, Moy M, Murphy B, Murphy L, Muzny DM, Nelson DL, Nelson DR, Nelson KA, Nixon K, Nusskern DR, Pacleb JM, Palazzolo M, Pittman GS, Pan S, Pollard J, Puri V, Reese MG, Reinert K, Remington K, Saunders RD, Scheeler F, Shen H, Shue BC, Siden-Kiamos I, Simpson M, Skupski MP, Smith T, Spier E, Spradling AC, Stapleton M, Strong R, Sun E, Svirskas R, Tector C, Turner R, Venter E, Wang AH, Wang X, Wang ZY, Wassarman DA, Weinstock GM, Weissenbach J, Williams SM, WoodageT, Worley KC, Wu D, Yang S, Yao QA, Ye J, Yeh RF, Zaveri JS, Zhan M, Zhang G, Zhao Q, Zheng L, Zheng XH, Zhong FN, Zhong W, Zhou X, Zhu S, Zhu X, Smith HO, Gibbs RA, Myers EW, Rubin GM, Venter JC: The genome sequence of Drosophila melanogaster. Science 2000;287:2185-2195. 
Dossena et al.: A Genetic Tool to Identify Functionally Related Proteins

6 Stumpf MP, Thorne T, de Silva E, Stewart R, An HJ, Lappe M, Wiuf C: Estimating the size of the human interactome. Proc Natl Acad Sci U S A 2008;105:6959-6964.

7 Furst J, Botta G, Saino S, Dopinto S, Gandini R, Dossena S, Vezzoli V, Rodighiero S, Bazzini C, Garavaglia ML, Meyer G, Jakab M, Ritter M, Wappl-Kornherr E, Paulmichl M: The ICln interactome. Acta Physiol (Oxf) 2006;187:43-49.

8 Khan SH, Ahmad F, Ahmad N, Flynn DC, Kumar R: Protein-protein interactions: principles, techniques, and their potential role in new drug development. J Biomol Struct Dyn 2011;28:929-938.

-9 Shekhawat SS, Ghosh I: Split-protein systems: Beyond binary protein-protein interactions. Current Opin Chem Biol 2011;15:789-797.

10 Gerstein MB, Bruce C, Rozowsky JS, Zheng D, Du J, Korbel JO, Emanuelsson O, Zhang ZD, Weissman S, Snyder M: What is a gene, post-ENCODE? History and updated definition. Genome Res 2007;17:669-681.

11 Skrabanek L, Saini HK, Bader GD, Enright AJ: Computational prediction of protein-protein interactions. Mol Biotechnol 2008;38:1-17.

12 Lees JG, Heriche JK, Morilla I, Ranea JA, Orengo CA: Systematic computational prediction of protein interaction networks. Phys Biol 2011;8:035008.

13 Ferrer L, Dale JM, Karp PD: A systematic study of genome context methods: calibration, normalization and combination. BMC Bioinformatics 2010;11:493.

14 Grigoriev A: A relationship between gene expression and protein interactions on the proteome scale: alysis of the bacteriophage T7 and the yeast Saccharomyces cerevisiae. Nucleic Acids Res 2001;29:3513-3519.

15 Ge H, Liu Z, Church GM, Vidal M: Correlation between transcriptome and interactome mapping data from Saccharomyces cerevisiae. Nat Genet 2001;29:482-486.

16 Teichmann SA, Babu MM: Conservation of gene co-regulation in prokaryotes and eukaryotes. Trends Biotechnol 2002;20:407-410; discussion 410.

$\checkmark 17$ Jansen R, Greenbaum D, Gerstein M: Relating whole-genome expression data with protein-protein interactions. Genome Res 2002;12:37-46.

-18 Dandekar T, Snel B, Huynen M, Bork P: Conservation of gene order: a fingerprint of proteins that physically interact. Trends Biochem Sci 1998;23:324-328.

19 Blumenthal T: Operons in eukaryotes. Brief Funct Genomic Proteomic 2004;3:199-211.

20 Overbeek R, Fonstein M, D'Souza M, Pusch GD, Maltsev N: The use of gene clusters to infer functional coupling. Proc Natl Acad Sci U S A 1999;96:2896-2901.

21 Snel B, Bork P, Huynen MA: The identification of functional modules from the genomic association of genes. Proc Natl Acad Sci U S A 2002;99:5890-5895.

22 Blumenthal T, Evans D, Link CD, Guffanti A, Lawson D, Thierry-Mieg J, Thierry-Mieg D, Chiu WL, Duke K, Kiraly M, Kim SK: A global analysis of Caenorhabditis elegans operons. Nature 2002;417:851-854.

23 Allen MA, Hillier LW, Waterston RH, Blumenthal T: A global analysis of C. elegans trans-splicing. Genome Res 2011;21:255-264.

-24 Blumenthal T, Gleason KS: Caenorhabditis elegans operons: form and function. Nat Rev Gen 2003;4:112120.

-25 Morton JJ, Blumenthal T: Identification of transcription start sites of trans-spliced genes: uncovering unusual operon arrangements. RNA 2011;17:327-337.

-26 Huang P, Pleasance ED, Maydan JS, Hunt-Newbury R, O'Neil NJ, Mah A, Baillie DL, Marra MA, Moerman DG, Jones SJ: Identification and analysis of internal promoters in Caenorhabditis elegans operons. Genome Res 2007;17:1478-1485.

27 Blumenthal T: Trans-splicing and operons. WormBook 2005:1-9.

28 Blumenthal T: Trans-splicing and operons in C. elegans. WormBook 2012:1-11.

29 Hillier LW, Reinke V, Green P, Hirst M, Marra MA, Waterston RH: Massively parallel sequencing of the polyadenylated transcriptome of $C$. elegans. Genome Res 2009;19:657-666.

30 Blumenthal T: Trans-splicing and polycistronic transcription in Caenorhabditis elegans. Trends Genet 1995;11:132-136.

-31 Osbourn AE, Field B: Operons. Cell Mol Life Sci 2009;66:3755-3775.

32 Guiliano DB, Blaxter ML: Operon conservation and the evolution of trans-splicing in the phylum Nematoda. PLoS Genet 2006;2:e198. 
Dossena et al.: A Genetic Tool to Identify Functionally Related Proteins

33 Furst J, Ritter M, Rudzki J, Danzl J, Gschwentner M, Scandella E, Jakab M, Konig M, Oehl B, Lang F, Deetjen P, Paulmichl M: ICln ion channel splice variants in Caenorhabditis elegans: voltage dependence and interaction with an operon partner protein. J Biol Chem 2002;277:4435-4445.

-34 Eichmuller S, Vezzoli V, Bazzini C, Ritter M, Furst J, Jakab M, Ravasio A, Chwatal S, Dossena S, Botta G, Meyer G, Maier B, Valenti G, Lang F, Paulmichl M: A new gene-finding tool: using the Caenorhabditis elegans operons for identifying functional partner proteins in human cells. J Biol Chem 2004;279:71367146.

-35 Dossena S, Gandini R, Tamma G, Vezzoli V, Nofziger C, Tamplenizza M, Salvioni E, Bernardinelli E, Meyer G, Valenti G, Wolf-Watz M, Furst J, Paulmichl M: The molecular and functional interaction between ICln and HSPC038 proteins modulates the regulation of cell volume. J Biol Chem 2011;286:40659-40670. Williams K: Interactions of polyamines with ion channels. Biochem J 1997;325:289-297.

Uehara A, Fill M, Velez P, Yasukochi M, Imanaga I: Rectification of rabbit cardiac ryanodine receptor current by endogenous polyamines. Biophys J 1996;71:769-777.

-38 Palade P: Drug-induced Ca2+ release from isolated sarcoplasmic reticulum. III. Block of Ca2+-induced Ca2+ release by organic polyamines. J Biol Chem 1987;262:6149-6154.

-39 Jospin M, Qi YB, Stawicki TM, Boulin T, Schuske KR, Horvitz HR, Bessereau JL, Jorgensen EM, Jin Y: A neuronal acetylcholine receptor regulates the balance of muscle excitation and inhibition in Caenorhabditis elegans. PLoS Biol 2009;7:e1000265.

40 Groot-Kormelink PJ, Boorman JP, Sivilotti LG: Formation of functional alpha3beta4alpha5 human neuronal nicotinic receptors in Xenopus oocytes: a reporter mutation approach. Br J Pharmacol 2001;134:789-796.

41 Wang F, Gerzanich V, Wells GB, Anand R, Peng X, Keyser K, Lindstrom J: Assembly of human neuronal nicotinic receptor alpha5 subunits with alpha3, beta2, and beta4 subunits. J Biol Chem 1996;271:1765617665.

42 Improgo MR, Scofield MD, Tapper AR, Gardner PD: The nicotinic acetylcholine receptor CHRNa5/a3/b4 gene cluster: dual role in nicotine addiction and lung cancer. Prog Neurobiol 2010;92:212-226.

43 Lipkowitz S, Gobel V, Varterasian ML, Nakahara K, Tchorz K, Kirsch IR: A comparative structural characterization of the human NSCL-1 and NSCL-2 genes. Two basic helix-loop-helix genes expressed in the developing nervous system. J Biol Chem 1992;267:21065-21071.

44 Mattar P, Britz O, Johannes C, Nieto M, Ma L, Rebeyka A, Klenin N, Polleux F, Guillemot F, Schuurmans C: A screen for downstream effectors of neurogenin2 in the embryonic neocortex. Dev Biol 2004;273:373-389.

45 Schuurmans C, Armant O, Nieto M, Stenman JM, Britz O, Klenin N, Brown C, Langevin LM, Seibt J, Tang H, Cunningham JM, Dyck R, Walsh C, Campbell K, Polleux F, Guillemot F: Sequential phases of cortical specification involve neurogenin-dependent and -independent pathways. EMBO J 2004;23:2892-2902.

-46 Derbyshire ER, Marletta MA: Structure and regulation of soluble guanylate cyclase. Annu Rev Biochem 2012;81:533-559.

47 Garthwaite J, Southam E, Anderton M: A kainate receptor linked to nitric oxide synthesis from arginine. J Neurochem 1989;53:1952-1954.

48 Rodrigo R, Montoliu C, Chatauret N, Butterworth R, Behrends S, Del Olmo JA, Serra MA, Rodrigo JM, Erceg $\mathrm{S}$, Felipo V: Alterations in soluble guanylate cyclase content and modulation by nitric oxide in liver disease. Neurochem Int 2004;45:947-953.

49 Montoliu C, Rodrigo R, Monfort P, Llansola M, Cauli O, Boix J, Elmlili N, Agusti A, Felipo V: Cyclic GMP pathways in hepatic encephalopathy. Neurological and therapeutic implications. Metab Brain Dis 2010;25:39-48.

50 Treinin M, Gillo B, Liebman L, Chalfie M: Two functionally dependent acetylcholine subunits are encoded in a single Caenorhabditis elegans operon. Proc Natl Acad Sci U S A 1998;95:15492-15495.

51 Treinin M, Chalfie M: A mutated acetylcholine receptor subunit causes neuronal degeneration in C. elegans. Neuron 1995;14:871-877.

52 Adcock C, Smith GR, Sansom MS: The nicotinic acetylcholine receptor: from molecular model to singlechannel conductance. Eur Biophys J 2000;29:29-37.

53 Furst J, Jakab M, Konig M, Ritter M, Gschwentner M, Rudzki J, Danzl J, Mayer M, Burtscher CM, Schirmer J, Maier B, Nairz M, Chwatal S, Paulmichl M: Structure and function of the ion channel ICln. Cell Physiol Biochem 2000;10:329-334.

54 Pu WT, Wickman K, Clapham DE: ICln is essential for cellular and early embryonic viability. J Biol Chem 2000;275:12363-12366. 
55 Paulmichl M, Li Y, Wickmann K, Ackerman M, Peralta E, Clapham D: New mammalian chloride channel identified by expression cloning. Nature 1992;356:238-241.

-56 Ritter M, Ravasio A, Jakab M, Chwatal S, Furst J, Laich A, Gschwentner M, Signorelli S, Burtscher C, Eichmuller S, Paulmichl M: Cell swelling stimulates cytosol to membrane transposition of ICln. J Biol Chem 2003;278:50163-50174.

57 Lang F, Shumilina E, Ritter M, Gulbins E, Vereninov A, Huber SM: Ion channels and cell volume in regulation of cell proliferation and apoptotic cell death. Contrib Nephrol 2006;152:142-160.

58 Tamma G, Procino G, Strafino A, Bononi E, Meyer G, Paulmichl M, Formoso V, Svelto M, Valenti G: Hypotonicity induces aquaporin-2 internalization and cytosol-to-membrane translocation of ICln in renal cells. Endocrinology 2007;148:1118-1130.

59 Gandini R, Dossena S, Vezzoli V, Tamplenizza M, Salvioni E, Ritter M, Paulmichl M, Furst J: LSm4 associates with the plasma membrane and acts as a co-factor in cell volume regulation. Cell Physiol Biochem 2008;22:579-590.

60 Spieth J, Brooke G, Kuersten S, Lea K, Blumenthal T: Operons in C. elegans: Polycistronic mRNA precursors are processed by trans-splicing of SL2 to downstream coding regions. Cell 1993;73:521-532.

61 Coronado R, Latorre R: Phospholipid bilayer made from monolayers on patch-clamp pipettes. Biophys J 1983;43:231-236.

62 Blumenthal T: Gene cluster and polycistronic transcription in eukaryotes. BioEssay 1998;20:480-487.

63 Ben-Shahar Y, Nannapaneni K, Casavant TL, Scheetz TE, Welsh MJ: Eukaryotic operon-like transcription of functionally related genes in Drosophila. Proc Natl Acad Sci U S A 2007;104:222-227.

64 Pi H, Lee LW, Lo SJ: New insights into polycistronic transcripts in eukaryotes. Chang Gung Med J 2009;32:494-498.

65 Ben-Shahar Y, Lu B, Collier DM, Snyder PM, Schnizler M, Welsh MJ: The Drosophila gene Cheb42a is a novel modifier of Deg/ENaC channel function. PLoS One 2010;5:e9395.

66 Lee SJ: Expression of growth/differentiation factor 1 in the nervous system: conservation of a bicistronic structure. Proc Natl Acad Sci U S A 1991;88:4250-4254.

67 Wang B, Shi G, Fu Y, Xu X: Cloning and characterization of a LASS1-GDF1 transcript in rat cerebral cortex: conservation of a bicistronic structure. DNA Seq 2007;18:92-103.

68 Derer S, Till A, Haesler R, Sina C, Grabe N, Jung S, Nikolaus S, Kuehbacher T, Groetzinger J, Rose-John S, Rosenstiel PC, Schreiber S: Mtnf reverse signalling induced by TNFalpha antagonists involves a GDF-1 dependent pathway: implications for crohn's disease. Gut 2013;62:376-386.

69 Gray TA, Saitoh S, Nicholls RD: An imprinted, mammalian bicistronic transcript encodes two independent proteins. Proc Natl Acad Sci U S A 1999;96:5616-5621.

70 Nicholls RD, Saitoh S, Horsthemke B: Imprinting in Prader-Willi and Angelman syndromes. Trends Genet 1998;14:194-200.

71 Reiss J, Cohen N, Dorche C, Mandel H, Mendel RR, Stallmeyer B, Zabot MT, Dierks T: Mutations in a polycistronic nuclear gene associated with molybdenum cofactor deficiency. Nat Genet 1998;20:51-53.

72 Rajagopalan KV, Johnson JL: The pterin molybdenum cofactors. J Biol Chem 1992;267:10199-10202.

-73 Reiss J, Dorche C, Stallmeyer B, Mendel RR, Cohen N, Zabot MT: Human molybdopterin synthase gene: Genomic structure and mutations in molybdenum cofactor deficiency type b. Am J Hum Genet 1999;64:706-711.

74 Gray TA, Nicholls RD: Diverse splicing mechanisms fuse the evolutionarily conserved bicistronic MOCS1a and MOCS1b open reading frames. RNA 2000;6:928-936. 